\title{
Beyond freeze-drying of biologics: vacuum-foam drying and spray freeze-drying
}

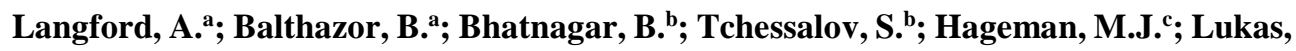
A. ; Plitzko, M. ${ }^{\mathrm{d}}$; Luy, B $^{\mathrm{d}}$; Ohtake, S. ${ }^{\mathrm{a}^{*}}$

${ }^{\text {a }}$ Pfizer, Inc. BioTherapeutics Pharmaceutical Sciences, Chesterfield, MO, USA.

b Pfizer, Inc. BioTherapeutics Pharmaceutical Sciences, Andover, MA, USA.

${ }^{\text {c }}$ University of Kansas, Department of Pharmaceutical Chemistry, Lawrence, KS, USA.

${ }^{\mathrm{d}}$ Meridion Technologies GmbH, Müllheim, Germany.

*E-mail of the corresponding author: Satoshi.ohtake@pfizer.com

\begin{abstract}
The complexity of biotherapeutics in development continues to increase as our capability in discovery and recombinant technology improves. While safety and efficacy remain the two critical aspects of all therapeutics, ensuring adequate stability is a challenge. Freeze-drying is a commonly-used processing technique to enhance the stability of biotherapeutic products, although the lengthy process time and low energy efficiency have led to the search for, and evaluation of, next-generation drying technologies, including spray freezedrying and vaccum-foam drying. Both processes result in dosage forms that vary considerably from those produced by lyophilization and possess physical properties that may be deemed superior for their intended applications.
\end{abstract}

Keywords: vacuum-foam drying; spray freeze-drying; lyophilization; biotherapeutics; stabilization 


\section{Introduction}

Most biological materials contain high water content (typically $\geq 80 \% \mathrm{w} / \mathrm{w}$ ). Removal of water through drying provides numerous benefits, including ease of handling and storage, reduction in transportation costs, and improved stability, to name a few. Though all drying techniques share a common objective (i.e., dehydration), conceptually they are different and require modification/adaptation based on the properties of the compound.

Numerous commercially-approved products are manufactured by freeze-drying,[1] thus lyophilization represents the gold standard to which novel drying methods must be compared. Despite its prevalent use, novel technologies are continuously being evaluated, including vacuum foam-drying and spray freeze-drying, as will be described herein. Furthermore, there are a great number of drying technologies that are available, if not already in use, in the food, agriculuture, and textile industries.[2] As the sensitivity of pharmaceuticals is unique to the given compound, the selected drying technique may not be universally applicable. By understanding the drying mechanism and the stresses involved, the drying techniques can be tailored for effective use.

\section{Materials and Methods}

\subsection{Vacuum-Foam Drying}

\subsubsection{T cell sample preparation}

Primary human pan-T cells (STEMCELL Technologies Inc, Cambridge, MA) were expanded 3-4 fold and cryopreserved at $-150^{\circ} \mathrm{C}$. During formulation, frozen T cells were thawed at ambient temperature, centrifuged, supernatant removed, and resuspended in the appropriate formulation matrix.

\subsubsection{Cell viability and count}

Viability measurements were obtained using a NucleoCounter ${ }^{\circledR} 3000$ (ChemoMetec A/S, Allerød, DK). Neat samples were diluted 4:1 in PBS and cell viability and count assay was performed using a Vai1-Cassette ${ }^{\mathrm{TM}}$. The procedure used membrane penetrating acridine orange (AO) and non-penetrating 4',6-diamidino-2-phenylindole (DAPI) fluorescent dyes to assess cellular membrane integrity. Dried samples were allowed to recover for 3 hours following reconstitution and diluted in PBS prior to analysis.

\subsubsection{Vacuum-Foam Drying and Freeze-Drying}

Vacuum-foam drying and freeze-drying were performed using LyoStar lyophilizers (SP Scientific, Warminster, PA). Vacuum-foam drying cycles utilized pressures and shelf 
temperatures ranging from 0.05 to 5 Torr and $5-30^{\circ} \mathrm{C}$, respectively. The freeze-drying cycle utilized $-30^{\circ} \mathrm{C}$ shelf temperature for primary drying at a pressure of 0.05 Torr.

\subsection{Spray Freeze-Drying}

Two process equipment types were utilized for spray freeze-drying. The first process step, spray freezing, was conducted at ambient pressure in a spray freezing chamber and the subsequent dynamic freeze drying of the frozen bulk was perfomed in a rotary bulk freeze dryer.

\subsubsection{Spray Freezing}

Spray Freezing in all scales was performed in a spray freezing chamber unit (SprayCon, Meridion, Germany) with frequency driven droplet formation nozzle(s) placed in the top lid. The droplet formation is achieved by controlled laminar jet break up. The cylindrical process chamber is double walled and cooled with gaseous and liquid nitrogen.

For all trials, the spray liquid is a $20 \%$ (w/w) sucrose solution, and a $300 \mu \mathrm{m}$ orifice opening for the nozzle was used. For the freezing step, the main process parameters are: (i) for lab and pilot scale trials: - $150{ }^{\circ} \mathrm{C}$ gas temperature; spray rate: $19 \mathrm{~g} / \mathrm{min}$ (1 nozzle); droplet size $550 \pm 10 \mu \mathrm{m}$; (ii) for commercial scale trial: - $120^{\circ} \mathrm{C}$ gas temperature; spray rate: $26 \mathrm{~g} / \mathrm{min} /$ nozzle (3 nozzles), droplet size $550 \pm 10 \mu \mathrm{m}$

For lab and pilot scale trials, the process equipment used was a stand-alone equipment with intermediate frozen storage of material at $-60^{\circ} \mathrm{C}$. For commercial scale, the trial was conducted in a fully contained process line that integrates both the spray freezing equipment and the rotary freeze dryer; e.g., the freezing chamber continously discharges the frozen spheres into the precooled drum of the rotary freeze dryer.

\subsubsection{Dynamic Freeze-Drying}

Lyophilization of the frozen sucrose pellets was performed in three different scales of rotary freeze dryer (RFD) equipment (all by Meridion, Germany); RFD LyoMotion LAB (lab scale), LyoMotion 30 (pilot scale), and LyoMotion 200 (commercial scale). All scales used a rotating, double walled drum which was positionesd in a vacuum process chamber, to which a condenser was attached. The drum temperature was controlled in a range from $-55^{\circ} \mathrm{C}$ up to $+50{ }^{\circ} \mathrm{C}$.

In all trials, sublimation energy was conveyed by contact heat, via the double wall of the rotating drum, and by infrared radiation emitted from one or more of the infrared sources that were positioned inside the drum above the moving bulk product surface. The pressure within the drying drum was maintained between 50 and $100 \mu$ bar at all three scales. 


\section{Results and Discussion}

\subsection{Vacuum-Foam Drying}

Vacuum-foam drying (VFD) transforms a solution or suspension into a dried static foam through a vacuum-induced evaporation and boiling process. VFD enables removal of water at low temperatures, which is required for heat labile biotherapeutics, through the use of a strong vacuum (e.g., 1 -10 Torr). For pharmaceutical applications, VFD can be performed using a lyophilizer capable of pressure control at a higher range than a typical freeze-drying (FD) cycle. In VFD, the boiling process results in a final product that has an expanded foam structure. Fig. 1 demonstrates that increasing sucrose concentration from 15 to $40 \%$ (w/v), while maintaining a $1 \mathrm{~mL}$ fill, correlated with increasing foam volumes in the final product. The reproducibility and heterogeneity of a VFD product appearance is a challenge that should be considered during formulation and process development.

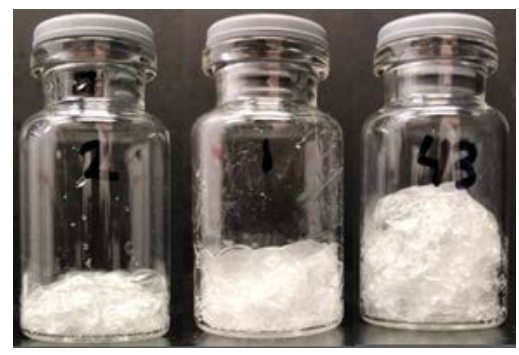

Fig 1 Vacuum-foam dried (VFD) preparations of 15\%, 30\%, and 40\% sucrose (left to right).

Compared to other drying techniques, dry static foams have been reported to provide significant stabilization to biotherapeutics. For example, Abdul-Fattah and coauthors [3, 4] have demonstrated improved storage stability of a monoclonal antibody and live virus vaccine as a dried foam in comparison to those prepared by spray drying and freeze drying. Currently, the use of cryopreservation techniques are required to stabilize cell-based therapeutics since the health of cellular suspensions decreases over a short period of time. The ability to stabilize mammalian cells in the dried state may reduce the logistical challenges of a supply chain for therapeutics requiring extremely low storage temperatures.

The $\mathrm{T}$ cells used in this work are primary human pan- $\mathrm{T}$ cells that were stored at $-150^{\circ} \mathrm{C}$ prior to preparation. T cell formulations evaluated include CryoStor ${ }^{\circledR}$ freeze media (with 0,5 or $10 \%$ DMSO) and disaccharide-based formulations (20\% sucrose/trehalose in PBS at pH 7.4) at $1 \mathrm{E} 6$ cells $/ \mathrm{mL}$ with $1 \mathrm{mg} / \mathrm{mL}$ bovine serum albumin. CyroStor freeze media is a commercially available preservation medium utilized for cryopreservation of cells at multiple concentrations of DMSO. Disaccharide-based formulations have been reported to provide significant stabilization to mammalian cells though the drying process and in the dried state 
$[5,6]$. Viability of pre- and post-dried preparations were measured using a NucleoCounter ${ }^{\circledR}$ 3000 fluorescent cell counting and membrane integrity assay.

Prior to drying, viability of all formulations was measured and no significant difference was observed. The average viability of the liquid controls was $91 \pm 3 \%$ (Fig. 2). All formulations were vacuum-foam dried using a fixed shelf temperature of $30{ }^{\circ} \mathrm{C}$ and the pressure was incrementally decreased from 5 to 0.05 Torr with a total drying time of 90 minutes. Drying was completed prior to an extended secondary drying step to minimize dehydration stress which could lead to additional viability loss. Fig. 2 presents the membrane integrity of T cell formulations following vacuum-foam drying and reconstitution with water. The viability of $\mathrm{T}$ cells after drying in CryoStor media was 65\%, with the addition of 5 and $10 \%$ DMSO resulting in post-drying viability of 57 and $32 \%$, respectively. The $20 \%$ sucrose and trehalose formulations resulted in post-drying viability of 63 and 65\%, respectively. While greater than $60 \%$ T cell viability was retained after drying various DMSO-free formulations, the recovery could be improved further through optimization of formulation, drying process parameters, and residual water content.[2]

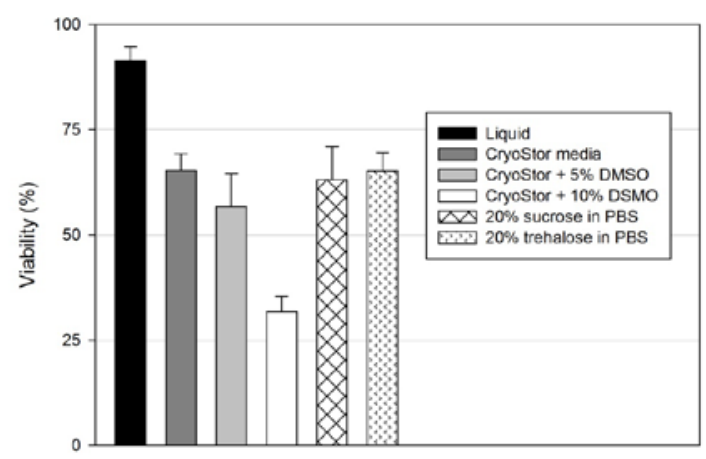

Fig. 2 Viability of human pan-T cell formulations after vacuum-foam drying compared to liquid controls. Data presented as average $\pm S D$.

A follow-up evaluation compared the storage stability of 7E5 T cells/mL formulated in 30\% trehalose and 3\% BSA in PBS at pH 7.4 as liquid, freeze-dried and foam dried preparations at $5^{\circ} \mathrm{C}$ (Fig. 3). The freeze and vacuum-foam drying cycles were designed to target a residual water content of $9 \%$ (w.b.). There was a higher post-drying viability for the VFD preparation compared to FD, for which the process loss was 61 and 42\% after FD and VFD, respectively. In order to decouple processing stress and storage stability, the viability results were normalized based on initial stability samples (post-drying). As shown in Fig. 3, the VFD preparation exhibited superior stability compared to liquid and FD preparations. At the same residual water content as a FD cake, these data provide evidence that $\mathrm{T}$ cells vitrified as a dried foam provides improved storage stability. 


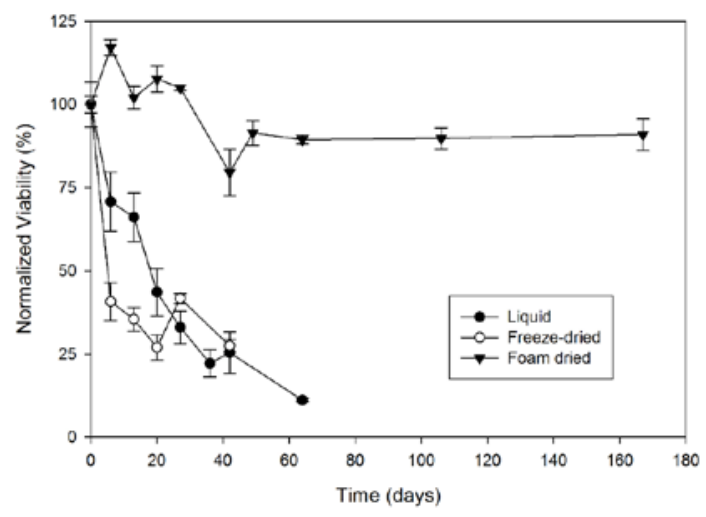

Fig. 3 Normalized viability of a liquid, freeze-dried, and foam dried preparations of $T$ cells on stability at $5^{\circ} \mathrm{C}$. Data presented as average $\pm 83 \% \mathrm{CI}$.

\subsection{Spray Freeze-Drying}

Spray freezing is often described as a process whereby the liquid droplets generated by spray nozzles (e.g., binary air nozzles or pressure nozzles) are directed into a cryogenic medium, often liquid nitrogen.[7] In such a case, the suspended frozen droplets are collected either by sieves or are collected after the cryogen has boiled off. For the spray freeze-drying technology presented herein, two unique processes have been developed and adapted for the use in the manufacture of parenteral pharmaceutical formulations.[8-10]

In the first process step, spray freezing, the frozen microspheres are generated as bulk by dispersing the substrate liquid into single droplets of homogeneous size,[11] utilizing a frequency nozzle, which fall through the cold gas (serving as cryogenic medium) and congeal to form frozen spheres. In contrast, the use of conventional spray nozzles results in a broader range of particle size with a significant amount of fines. The direct use of liquid nitrogen $\left(\mathrm{LN}_{2}\right)$ is avoided to minimize internal mechanical stress encountered during freezing, in particular for large particles. The freezing chamber is therefore designed as a cylindrical, double walled column. The process takes place under ambient pressure conditions. Droplet size depends on parameters such as flow rate, frequency, viscosity (based on formulation and temperature), and orifice diameter; for the current application, a (selectable) range for the droplets between 300 to $1000 \mu \mathrm{m}$ was targeted. For scale-up, multiple nozzles were used, though the height of the freezing column remained unchanged.

The second process step is the freeze drying of the frozen bulk material. The dynamic lyophilization process conducted in a rotary freeze dryer provides process conditions that produce bulk product with high homogeneity (Fig. 4), while avoiding specific aspects of fluid bed processing.[12] Generally speaking, processing conditions such as pressure and temperature are quite comparable to parameters utilized in conventional freeze drying. There 
are some differences to be noted, for example: the large surface of the frozen bulk increases heat and mass transfer, which generally allows for shorter drying times. Furthermore, the water vapor diffusion length is significantly reduced. A $10 \mathrm{~mm}$ lyo cake in a vial poses a maximum diffusion length of $10,000 \mu \mathrm{m}$; in a $1 \mathrm{~mm}$ microsphere, the maximum length is $500 \mu \mathrm{m}$. In conventional shelf freeze drying, the heat to a large portion is conveyed across the bottom of the glass vial; the drying front is moving from the top to the bottom, i.e. the heat transfer takes place across the frozen product. In dynamic freeze drying, the energy both from radiators and drum surface is transmitted to the surface of a particle, at which the drying front initiates.

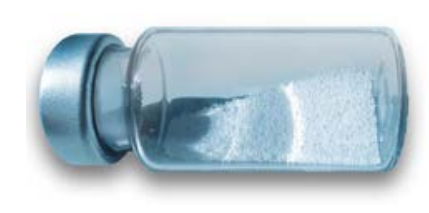

Fig. 4 Spray Freeze-Dried microspheres generated for a 20\% (w/w) sucrose solution.

The results for spray freeze-dried sucrose conducted at three scales are shown in Table 1. In all scales, residual water content less than $1 \%$ can be reached and that yield above $95 \%$ is possible in commercial scale. The lower yield in pilot scale is explained by the use of higher rpm of the drum in conjunctions with higher IR power. During drying, a pellet will loose 80$90 \%$ of its weight. High water vapor flows would cause the pellets to get entrapped into the vapor flow, which would cause particles to leave the drum, reducing the yield. Additional factors need to be considered, such as solid content, as higher solid content reduces the loss in weight, and electrostatic phenomena, which may be significant if the particle size is too low. The level of residual water content achieved is comparable to that from conventional freeze drying.

Table 1. Results for $20 \%(w / w)$ sucrose solution processed at three scales

\begin{tabular}{lccc} 
& Lab scale & Pilot scale & Commercial scale \\
\hline Amount processed (kg) & 1 & 6 & 107 \\
Drying time (h) & 5.5 & 16 & 29.25 \\
Yield (\%, w/w) & 98.6 & 81.6 & 97.3 \\
Residual water content (\%) & $\leq 1.0$ & $\leq 1.0$ & $\leq 0.6$ \\
Reconstitution time (min) & $\leq 1.0$ & $\leq 1.0$ & $\leq 1.0$ \\
\hline
\end{tabular}

\section{Conclusions}

The complexity of biotherapeutics in development continues to increase as our capability in discovery and recombinant technology improves. While safety and efficacy remain the two critical aspects of all therapeutics, stability, both in terms of shelf-life and to stresses encountered during manufacturing, remains a challenge. Spray freeze-drying is a hybrid 
processing technology comprising spray drying and bulk freeze-drying, while vacuum foam drying is a modified freeze-drying process that challenges the conventional processing conditions utilized in lyophilization. The former has matured to a level where the application of the technology even in commercial scale is in reach also for pharmaceutical applications, while the latter has provided enhanced stability to a complex biological beyond that provided by a conventional freeze-drying process. The development of novel drying technologies, such as the aforementioned processes, is a culmination of fundamental understanding gained in academia and leveraging the lessons learned through their utilization in orthogonal industries. For implementation, technical evaluation should include the scalability of the process, energy efficiency, as well as the capability to implement the technique in a GMP environment.

\section{Acknowledgements}

Karin Mayer for supporting the SFD lab and pilot work and Thomas Gebhard and Roland Kaiser for the commercial scale trial, all performed at Meridion, Germany.

\section{References}

[1] Costantino, H.R. Excipients for use in lyophilized pharmaceutical peptide, protein and other bioproducts. In Lyophilization of Biopharmaceuticals; Costantino, H.R., Pikal, M. J., Eds.; AAPS Press: Arlington, VA, 2004; pp 139-228.

[2] Walters, R. H.; Bhatnagar, B.; Tchessalov, S.; Izutsu, K. I.; Tsumoto, K.; Ohtake, S. Next generation drying technologies for pharmaceutical applications. J. Pharm. Sci. 2014. 103 (9):2673-2695.

[3] Abdul - Fattah, A. M.; Truong - Le, V.; Yee, L.; Nguyen, L.; Kalonia, D. S.; Cicerone, M. T.; Pikal, M. J. Drying-Induced Variations in Physico-Chemical Properties of Amorphous Pharmaceuticals and Their Impact on Stability (I): Stability of a Monoclonal Antibody. J. Pharm. Sci. 2007. 96 (8):1983-2008.

[4] Abdul-Fattah, A. M.; Truong-Le, V.; Yee, L.; Pan, E.; Ao, Y.; Kalonia, D. S.; Pikal, M. J. DryingInduced Variations in Physico-Chemical Properties of Amorphous Pharmaceuticals and Their Impact on Stability II: Stability of a Vaccine. Pharm. Res. 2007. 24 (4):715.

[5] Crowe, J. H.; Crowe, L. M.; Wolkers, W.; Tsvetkova, N. M.; Oliver, A.; Torok, Z.; Kheirolomoom, A.; Norris, J.; Satpathy, G.; Ma, X.; et al. Stabilization of Mammalian Cells in the Dry State. In Advances in Biopreservation; Baust, J. G., Baust, J. M., Eds.; CRC Press: Boca Raton, 2006; pp 383-411.

[6] Crowe, J. H.; Crowe, L. M.; Oliver, A. E.; Tsvetkova, N.; Wolkers, W.; Tablin, F. The trehalose myth revisited: introduction to a symposium on stabilization of cells in the dry state. Cryobiology 2001. 43 (2):89-105.

[7] Yu, Z.; Garcia, A. S.; Johnston, K. P.; Williams III, R. O. Spray freezing into liquid nitrogen for highly stable protein nanostructured microparticles. Eur. J. Pharm. Biopharm. 2004. 58 (3):529537.

[8] Luy, B.; Plitzko, M.; Struschka, M. 2016. Process line for the production of freeze-dried particles. EP2764309.

[9] Struschka, M.; Plitzko, M.; Gebhard, T.; Luy, B. 2016. Rotary drum for use in a vacuum freezedryer. EP2764310.

[10] Plitzko, M.; Struschka, M.; Gebhard, T.; Luy, B. 2015. A process line for the production of freezedried particles. EP2764311.

[11] Mishra, M., ed. Handbook of encapsulation and controlled release. CRC Press: Boca Raton, 2015.

[12] Plitzko, M. 2006. "Freeze-drying in the fluidized bed: Possibilities and limits for use in pharmacy." Department of Pharmaceutical Sciences, University of Basel. 